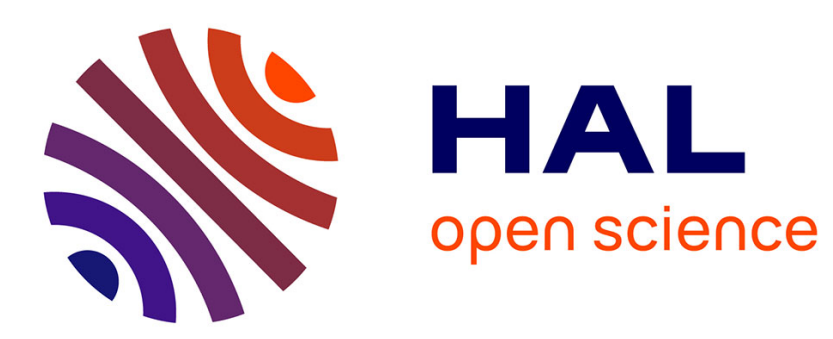

\title{
Excitation d'une résonance atomique par laser fluorescent à fréquence accordable
}

\author{
G. Megie, O. de Witte
}

\section{To cite this version:}

G. Megie, O. de Witte. Excitation d'une résonance atomique par laser fluorescent à fréquence accordable. Revue de Physique Appliquée, 1971, 6 (3), pp.341-343. 10.1051/rphysap:0197100603034100 . jpa-00243553

\section{HAL Id: jpa-00243553 https://hal.science/jpa-00243553}

Submitted on 1 Jan 1971

HAL is a multi-disciplinary open access archive for the deposit and dissemination of scientific research documents, whether they are published or not. The documents may come from teaching and research institutions in France or abroad, or from public or private research centers.
L'archive ouverte pluridisciplinaire HAL, est destinée au dépôt et à la diffusion de documents scientifiques de niveau recherche, publiés ou non, émanant des établissements d'enseignement et de recherche français ou étrangers, des laboratoires publics ou privés. 


\title{
EXCITATION D'UNE RÉSONANCE ATOMIQUE PAR LASER FLUORESCENT A FRÉQUENCE ACCORDABLE
}

\author{
G. MEGIE \\ Service Aéronomie du C. N. R. S. \\ O. de WITTE \\ Groupe de Physique Moléculaire, Ecole Polytechnique \\ (Reçu le 28 janvier 1971, révisé le 22 mars 1971)
}

\begin{abstract}
Résumé. - L'emploi des lasers organiques à molécules fluorescentes à fréquence accordable est particulièrement bien adapté à l'excitation des résonances atomiques. On décrit un montage donnant une largeur de raie d'émission laser voisine de la largeur des raies $D_{1}$ et $D_{2}$ du sodium à $160^{\circ} \mathrm{C}$. On observe le spectre de résonance du sodium excité par des raies de $1 \AA$ et de $0,1 \AA$ de largeur à mi-hauteur.
\end{abstract}

Abstract. - Tunable dye lasers are specially well adapted to excite atomic resonant scattering. We describe an experiment giving a spectral laser line, almost as narrow as $\mathbf{D}_{1}$ and $\mathbf{D}_{2}$ sodium lines at $160^{\circ} \mathrm{C}$. The resonance spectrum of sodium excited by laser lines $1 \AA$ and $0.1 \AA$ large is given.

Introduction. - Les lasers à molécules organiques fluorescentes en solution [1], grâce à leurs propriétés de condensation spectrale [2], [3] sont des instruments particulièrement adaptés à l'excitation des transitions atomiques ou moléculaires [4], [5], [6]. La présente note décrit une expérience de résonance dans la vapeur de sodium où l'excitation est réalisée par un laser à Rhodamine $6 \mathrm{G}$ en solution aqueuse ou alcoolique à température ambiante. On améliore ainsi considérablement la précision de l'accord en longueur d'onde sur une raie de résonance ainsi que l'énergie et la divergence par rapport aux résultats précédemment publiés [7], [8], [9].

Dispositif expérimental. - 1) LASER (Fig. 1). Le laser fluorescent utilise comme milieu actif la Rhodamine $6 \mathrm{G}$ en solution, soit dans l'eau additionnée d'acétamide, soit dans l'éthanol. L'emploi de l'eau avec acétamide comme solvant améliore les qualités optiques du milieu amplificateur [10]. L'excitation optique est effectuée par une lampe à éclairs coaxiale au xénon [11] alimentée par un condensateur de $0,4 \mu \mathrm{F}$ sous $30 \mathrm{kV}$. Les miroirs du laser ont res-

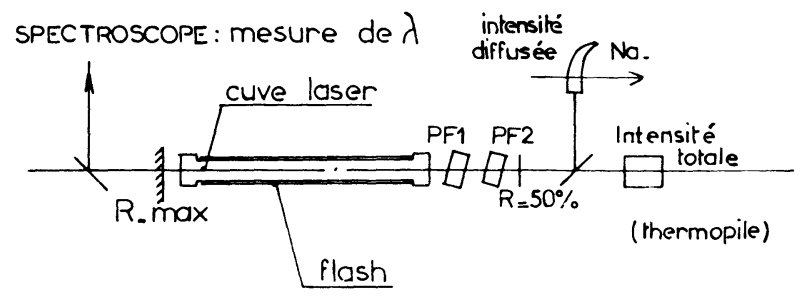

FIG. 1. - Schéma du montage expérimental. pectivement des coefficients de réflexion $>99 \%$ et $30 \%$. La solution laser circule en permanence afin d'améliorer l'homogénéité optique de la cavité et de refroidir la lampe à éclair, la cadence de tir est alors $0,1 \mathrm{~Hz}$.

Une énergie laser de $0,3 \mathrm{~J}$ est émise en cavité plan plan et sans aucun sélecteur spectral, sur une largeur de bande voisine de $120 \AA$ centrée sur $5900 \AA$. Pour étudier les transitions atomiques il est utile d'affiner cette bande. Le système de sélection spectrale est constitué d'un ou plusieurs Fabry-Pérot utilisés en transmission à l'intérieur de la cavité optique et inclinés sur l'axe laser.

Avec 3 Fabry-Pérot dont les intervalles entre ordres vont en décroissant on peut ne sélectionner qu'une seule raie. Le facteur de finesse des Fabry-Pérot varie entre 2,5 et $6(R=0,3$ et $R=0,6)$. Cette finesse est en fait multipliée par le nombre d'aller retour de l'onde laser dans la cavité, ainsi que par le phénomène de condensation spectrale due aux compétitions de modes.

La mesure de la largeur de raie laser obtenue est faite avec un Fabry-Pérot d'analyse de finesse $F>60$ et dont l'épaisseur est réglable. La divergence du faisceau est mesurée d'après la dimension de la tache laser sur un écran situé à une distance connue.

Les caractéristiques optiques sont les suivantes : tableau I.

On donne dans le tableau II les résultats obtenus avec et sans sélection spectrale pour une énergie de pompage de $150 \mathrm{~J}$. 


\section{TABLEAU I}

P. F. Ėpaisseur $\begin{gathered}\text { Intervalle } \\ \text { entre ordres } \\ \text { à } 0,6 \mu \mathrm{m}\end{gathered}$ Inclinaison

$\begin{array}{ccccc}- & - & - & - \\ \text { No 1 } & 15 \mu \mathrm{m} & 120 & \AA & 30 \mathrm{mrad} \\ \text { No 2 } & 100 \mu \mathrm{m} & 18 \AA & 50 \mathrm{mrad} \\ \text { No 3 } & 800 \mu \mathrm{m} & 2,2 \AA & 65 \mathrm{mrad}\end{array}$

résonance étant beaucoup plus fines $(\Delta \lambda=0,02 \AA)$ que la raie laser, la largeur de raie observée est en fait celle du laser. On retrouve bien la valeur de 1,5 mesurée précédemment avec le Fabry-Pérot d'analyse.

Le signal subsistant entre les deux pics d'absorption est dû aux réflexions parasites à l'intérieur de la cuve à résonance. On le vérifie aisément en mesurant le signal obtenu en cuve froide. La différence d'in-

TABLEAU II

$\begin{array}{lcccccc}\begin{array}{c}\text { Sélection } \\ \text { spectrale }\end{array} & \begin{array}{c}\text { Solution eau } \\ \text { Energie } \\ (\mathrm{mJ})\end{array} & \Delta \lambda(\AA) & \begin{array}{c}\text { Acétamide } 7,5 \times 10^{-5} \mathrm{~m} / 1 \\ \text { Divergence } \\ (\mathrm{mrad})\end{array} & \begin{array}{c}\text { Solution éthanol } 7,5 \times 10^{-5} \mathrm{~m} / 1 \\ \text { Energie } \\ (\mathrm{mJ})\end{array} & \Delta \lambda(\AA) & \begin{array}{c}\text { Divergence } \\ (\mathrm{mrad})\end{array} \\ \text { Sans P. F. } & - & - & - & - & - & - \\ \text { P. F. N } 1 & 200 & 120 & 2,5 & 200 & 110 & 4,5 \\ \text { P. F. Nos } 1,2 & 150 & 1,2 & 3 & 135 & 1,8 & 5 \\ \text { P. F. Nos } 1,2,3 & 100 & 0,15 & 3 & 85 & 0,28 & 5 \\ & 70 & 0,04 & 3,5 & 40 & 0,09 & 5\end{array}$

Afin de préciser l'influence des gradients d'indice $\mathrm{du}$ liquide sur les qualités optiques du laser on a également porté les résultats obtenus avec une solution d'éthanol.

2) Cuve a RÉSonANCE. - La cuve utilisée est une cellule de section carrée dont le queusot est coudé pour éliminer les réflexions parasites. La tête comporte deux " fenêtres » pour l'excitation et l'observation qui se font suivant deux directions perpendiculaires. Les fenêtres sont proches l'une de l'autre de façon à minimiser le chemin optique des photons de résonance. Afin d'éviter les dépôts métalliques sur les fenêtres, la tête et le queusot sont maintenus à deux températures différentes $T_{\text {tête }}=T_{\mathrm{q}}+20^{\circ}$. Ces deux températures sont maintenues constantes indépendamment au $1 / 10^{\mathrm{e}}$ de degré près. Pour une température du queusot de $145^{\circ} \mathrm{C}$ la densité d'atomes dans la cuve, fonction de la pression de vapeur saturante, est de l'ordre $10^{11}$ atomes. $\mathrm{cm}^{-3}$.

Le signal de résonance détecté à l'aide d'un photomultiplicateur est directement mesuré sur un oscilloscope. Il est ensuite corrigé en fonction de l'intensité laser à chaque tir afin d'éliminer les fluctuations possibles dans l'énergie émise.

Résultats obtenus en diffusion résonnante. - Nous avons tracé les courbes de résonance du sodium dans deux cas, avec le solvant aqueux.

a) Excitation avec Fabry-Pérot No 1 dans La CAVITÉ LASER. - Le balayage en longueur d'onde s'effectue par rotation du Fabry-Pérot. Le signal obtenu est représenté figure 2 . On voit apparaître deux bandes à 5890 et $5896 \AA$. Le signal ainsi observé donne le produit de convolution de la raie laser par la raie de résonance. Dans le cas présent les raies de

tensité entre les raies $\mathrm{D}_{2}$ et $\mathrm{D}_{1}$ correspond à la section efficace de résonance de $D_{2}$ double de celle de $D_{1}$ $\left(\sigma_{\mathrm{D}_{2}}=6,4 \times 10^{-12} \mathrm{~cm}^{2}\right)$

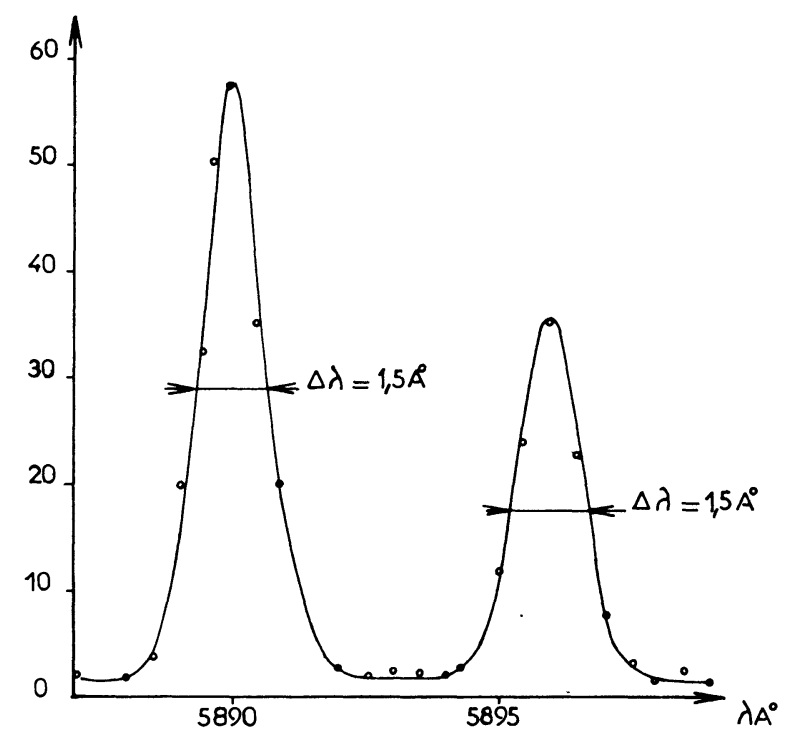

FIG. 2. - Intensité diffusée en fonction de $\lambda$ avec un seul FabryPérot dans la cavité.

b) ExCitation AVEC LeS Fabry-PÉRot No 1 eT $\mathrm{N}^{\circ} 2$ DANS LA CAVITÉ. - Le balayage en longueur d'onde s'effectue par rotation simultanée des deux Fabry-Pérot en ajustant à chaque fois les maximums de transmission.

La courbe (Fig. 3) donne ici une bien meilleure résolution spectrale des raies $D_{1}$ et $D_{2}$. La largeur de raie apparente est encore principalement due à la largeur de raie laser $(0,15 \AA)$, ce qui correspond bien à la largeur de raie mesurée expérimentalement par le Fabry-Pérot d'analyse. Il faut remarquer qu'en 


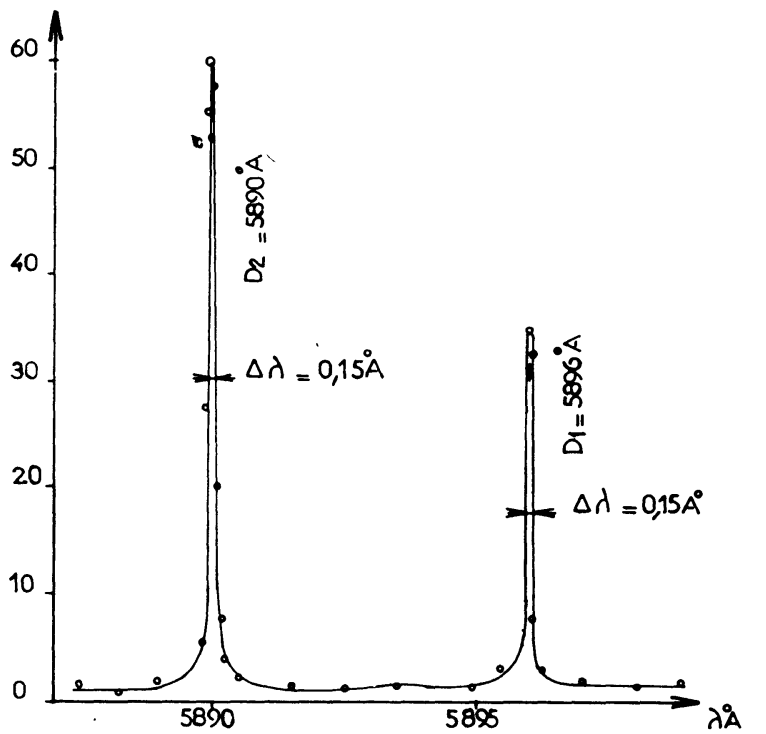

Fig. 3. - Intensité diffusée en fonction de $\lambda$ avec deux FabryPérot dans la cavité. dehors des raies de résonance du sodium, aucun signal autre que le signal dû aux réflexions parasites n'apparaît; la raie laser d'excitation est très bien définie sans épaulements, contrairement à ce qui se produit souvent avec un réseau comme sélecteur de longueur d'onde [3]. Cela est dû à la bonne résolution spectrale du Fabry-Pérot, même sous des inclinaisons de faisceau de quelques degrés.

Conclusion. - Les lasers à colorants fluorescents sont donc particulièrement bien adaptés à l'étude des transitions optiques fines du fait qu'il est possible de régler à la fois leur finesse de raie et leur longueur d'onde. L'emploi de 2 interféromètres Fabry-Pérot comme filtres de fréquence associés à un laser à colorants en solution aqueuse permet d'obtenir des énergies importantes $(0,1 \mathrm{~J})$ avec une faible divergence, et des caractéristiques spectrales bien définies $(\Delta \lambda \simeq 0,15 \AA)$ et stables.

\section{Bibliographie}

[1] Sorokin (P. P.), LANKaRd (J. R.), I. B. M., J. Res. Develop., 1966, 10, 162

[2] Soffer (B. H.), Macfarland (B. B.), Appl. Phys. Letters, 1967, 10, 266

[3] Bied-Charreton (P.) et al., C. R. Acad. Sci., Paris, $1969,238 \mathrm{~B}, 1377$.

[4] McIlrath (T. J.), Appl. Phys. Letters, 1969, 15, (2), 41.

[5] Yamaguchi (G.) et al., Japan, J. Appl. Phys., 1969, 8, 1265.

[6] Sorokin (P. P.), Lankard (J. R.), J. Chem. Phys., 1969, 51, (7).
[7] Gibson (A. J.), Journal of Scientific Instruments, 1969, 2, (2).

[8] Bonch Bruyevich (A. M.), Kostin (N. N.), AKhoDovor (V.), Optics and Spectroscopy, 1968, 24, (6), 547.

[9] Walther (H.), Hall (J. L.), Appl. Phys. Letters, 1970, 17, (6), 239.

[10] Crozet (P.), Meyer (Y.), C. R. Acad. Sci., Paris, 1970, $271 \mathrm{~B}, 718$.

[11] Furumoto (H. W.), Ceccon (H. L.), Appl. Optics, 1969, 8, (8), 1613. 